\title{
Polyphenol-Mediated Assembly for Particle Engineering
}

Jiajing Zhou, ${ }^{\dagger}$ Zhixing Lin, ${ }^{\dagger}$ Yi Ju, Md. Arifur Rahim, Joseph J. Richardson, and Frank Caruso* ARC Centre of Excellence in Convergent Bio-Nano Science and Technology, and the Department of Chemical Engineering, The University of Melbourne, Parkville, Victoria 3010, Australia

†J. Z. and Z. L. contributed equally

*Corresponding author. E-mail: fcaruso@unimelb.edu.au

\section{CONSPECTUS}

Polyphenols are naturally occurring compounds that are ubiquitous in plants and display a spectrum of physical, chemical, and biological properties. For example, they are antioxidants, have therapeutic properties, absorb UV radiation, and complex with metal ions. Additionally, polyphenols display high adherence, which has been exploited for assembling nanostructured materials. We previously reviewed the assembly of different phenolic materials and their applications (Angew. Chem. Int. Ed. 2019, 58, 1904-1927), however there is a need for a summary of the fundamental interactions that govern the assembly, stability and function of polyphenolbased materials. A detailed understanding of interactions between polyphenols and various other 
building blocks will facilitate the rational design and assembly of advanced polyphenol particles for specific applications.

This Account discusses how different physicochemical interactions (i.e., hydrogen bonds, $\pi$ interactions, hydrophobic effects, metal coordination, covalent bonds, and electrostatic interactions) can be leveraged to assemble and stabilize polyphenol-based particles for diverse applications. In polyphenol-mediated assembly strategies, the polyphenols typically exert more than one type of stabilizing attractive force. However, one interaction often dominates the assembly process and dictates the physicochemical behavior of the particles, which in turn influences potential applications. This Account is thus divided into sections that each focuses on a key interaction, with relevant examples of applications to highlight structure-function relationships. For example, metal coordination generally becomes weaker at lower $\mathrm{pH}$, which makes it possible to engineer metal-phenolic materials with a $\mathrm{pH}$-responsive disassembly profile suitable for drug delivery. Engineered particles, such as hollow capsules, mesoporous, and coreshell particles, and self-assembled nanoparticles are some of the systems that are covered to highlight how polyphenols interact with other building blocks, and therefore make up the major focus of this Account. Some of the applications of these materials exemplified in this Account include drug delivery, catalysis, environmental remediation, porous nanoparticle replication, and forensics. Finally, a perspective is provided on the current challenges and trends in polyphenolmediated particle assembly, and viable near-term strategies for further elucidating the interplay of various competing interactions in particle formation are discussed. This Account is also expected 
to serve as a reference to guide fundamental research and facilitate the rational design of polyphenol-based materials for diverse emerging applications.

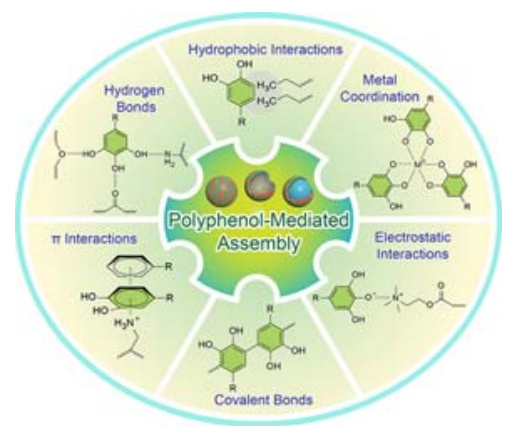




\section{INTRODUCTION}

Polyphenols are abundant, naturally occurring compounds, with over 8,000 unique molecules discovered to date. ${ }^{1}$ This abundance can be explained by the functional physical, chemical, and biological properties that polyphenols naturally convey to organisms, including UV absorption, metal ion complexation, anti-oxidation, and therapeutic efficacy. ${ }^{2}$ Therefore, polyphenols have been employed in traditional and modern medicine, tea and wine making, and other industries throughout history. ${ }^{3}$ Recently, there has been growing interest in polyphenol-based materials, including particles, films, and bulk hydrogels, owing to the discovery of the broad adherence arising from the catechol and gallol moieties in polyphenols. ${ }^{4}$ These chemical groups enable polyphenols to interact with diverse materials or substrates through a variety of interactions including hydrogen bonds, $\pi$ interactions, hydrophobic effects, metal coordination, covalent bonds, and electrostatic interactions. Therefore, polyphenols are widely compatible structural motifs for engineering materials for targeted applications spanning chemistry, materials science, and nanomedicine..$^{5,6}$

In the last decade, our group has explored a variety of polyphenol-mediated assemblies for materials engineering (Figure 1). ${ }^{7-19}$ In the present Account, the term "polyphenol" is used to refer to catechol- and gallol-containing aromatic building blocks with functionality common to polyphenols, without distinction between natural polyphenols and their synthetic derivatives. Specifically, we introduce the various interactions that polyphenols exhibit and how these can be used for particle assembly. The dominant interactions in each particle system give rise to unique physiochemical properties that complement the inherent functionality of the polyphenols 
themselves. We also discuss applications of the particles assembled through each specific interaction. Furthermore, we highlight challenges facing polyphenol-inspired materials and possible future research trends in this field.

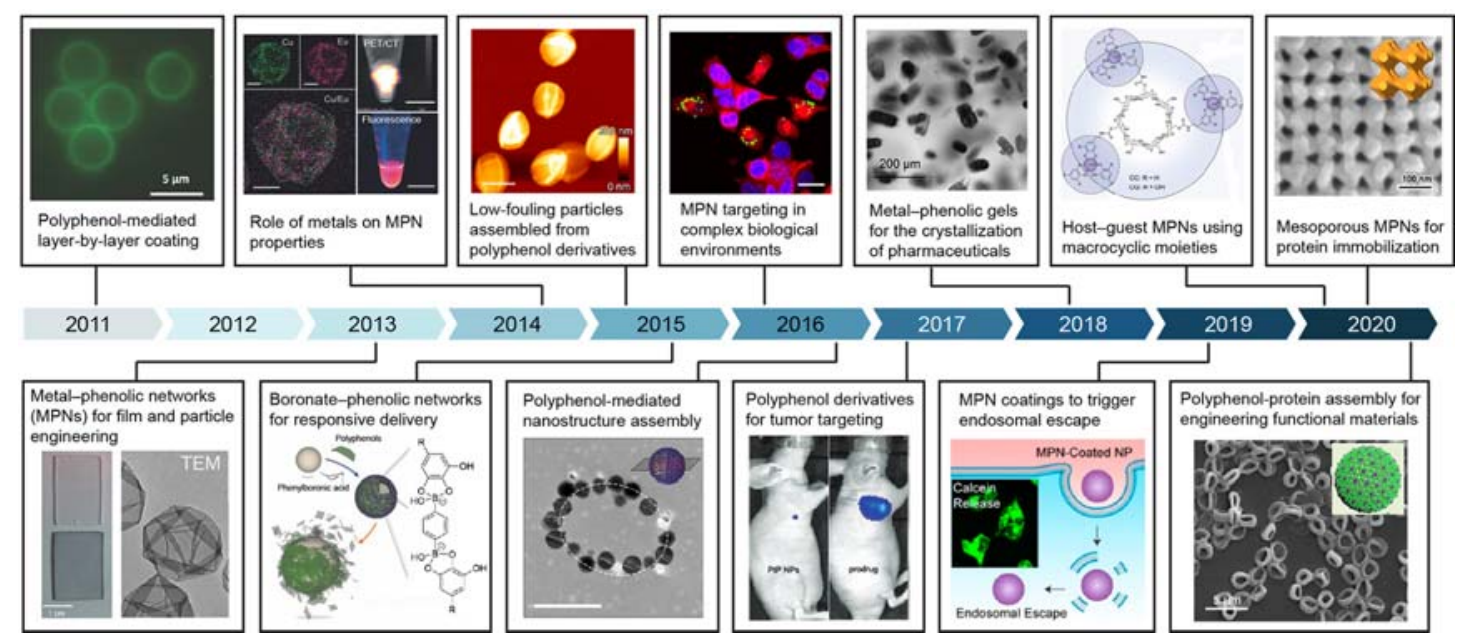

Figure 1. Timeline providing examples of polyphenol-based materials prepared from Caruso and co-workers. Adapted with permission from refs. 7-19. Copyright 2011, 2014, 2015, 2017, 2018, 2019, and 2020 Wiley-VCH. Copyright 2013 American Association for the Advancement of Science. Copyright 2015, 2016, 2019, and 2020 American Chemical Society. Copyright 2016 Springer Nature.

\section{POLYPHENOL INTERACTIONS}

Polyphenols are highly adherent molecules, as they interact with most materials owing to the hydrophobic, hydrophilic, and charge shifting regions of the catechol and gallol moieties. ${ }^{8,20}$ Figure 2 shows the structures of some typical natural polyphenols and the different key interactions that polyphenols can display with different materials. Many polyphenol-based particles are formed as a result of more than one type of stabilizing force but often one of the interactions dominates. 
The interactions dictate the physicochemical behavior of the particles, which in turn influences their potential applications. The following subsections thus focus on the key interactions of polyphenols and relevant examples of applications to demonstrate the structure-function relationship arising from careful choice of the building blocks.|
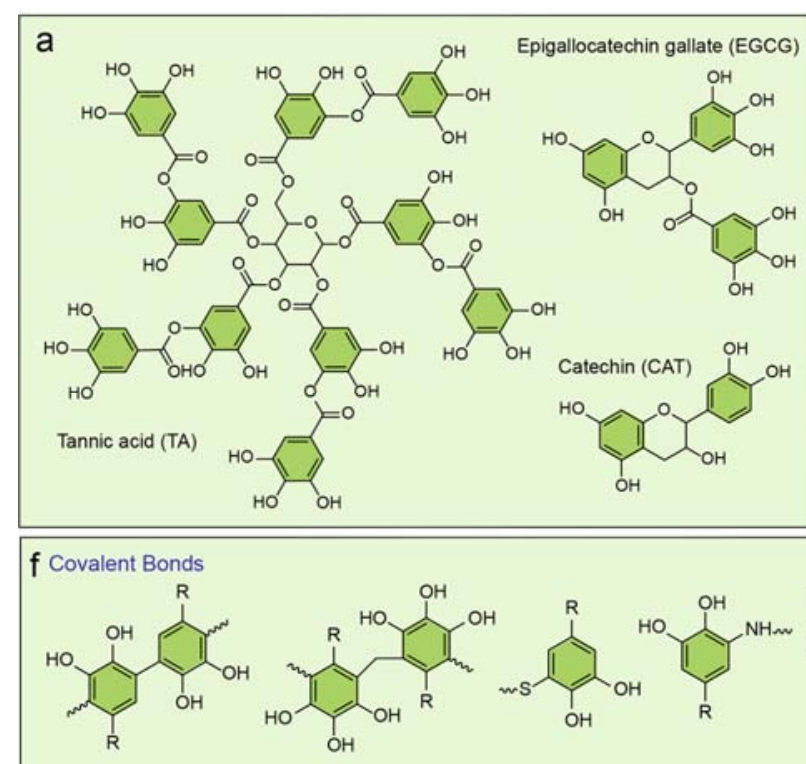

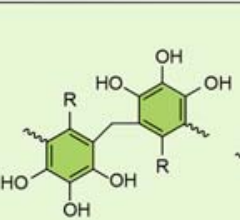

$\mathrm{OH}$

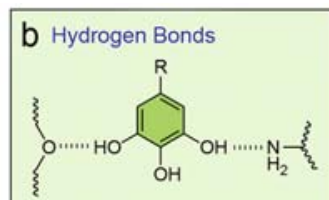

C Hydrophobic Interactions

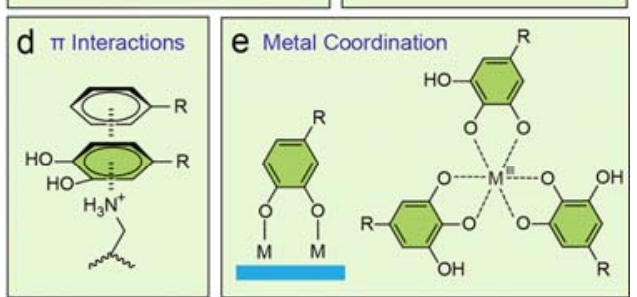

g Electrostatic Interactions

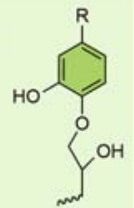

Figure 2. (a) Examples of natural polyphenol building blocks commonly used for synthesizing functional particles. (b-g) Polyphenols play a central role in engineering functional materials owing to their various interactions with different materials, as exemplified here.

\subsection{Hydrogen Bonds}

A hydrogen bond is typically regarded as a weak electrostatic attraction that occurs between a hydrogen atom (hydrogen-bond donor) and an electronegative atom such as nitrogen, oxygen, or fluorine (hydrogen-bond acceptor). It is one of the most frequently used dynamic bonds to assemble supramolecular networks with stimuli-responsive properties. The relatively high $\mathrm{p} K_{\mathrm{a}}$ of 
polyphenols (8.7 for epicatechin, ${ }^{21} 8.5$ for tannic acid (TA), ${ }^{22}$ and 7.7 for epigallocatechin-3-Ogallate $\left.(\mathrm{EGCG})^{21}\right)$ and their multiple protonation states make them strong hydrogen-bond donors at physiological $\mathrm{pH}$. For synthetic polymers, a phenolic hydroxyl group $(-\mathrm{OH})$ for example interacts with an ether group $(-\mathrm{O}-)$ in poly(ethylene glycol) $(\mathrm{PEG}),{ }^{23}$ a carbonyl group $(\mathrm{C}=\mathrm{O})$ in poly $\left(N\right.$-vinylpyrrolidone) $(\mathrm{PVPON}),{ }^{24}$ or an amine group $\left(-\mathrm{NH}_{2}\right)$ in poly(allylamine $) .{ }^{25}$

Polyphenols can also interact with biomacromolecules (e.g., polysaccharides, nucleic acids, and proteins) through the formation of multiple hydrogen bonds. For example, TA forms hydrogen bonds with the phosphate backbone of $\mathrm{DNA}^{26}$ or the carbonyl groups of peptide linkages. ${ }^{27}$ Hydrogen bonds are also involved in the interaction of polyphenols with various inorganic materials (e.g., mica). ${ }^{28}$ The $-\mathrm{OH}$ in polyphenols also sometimes serves as hydrogen-bond acceptors to form intramolecular hydrogen bonds depending on the bond dissociation enthalpy of the phenolic hydroxyl group and surrounding steric effects. ${ }^{29}$

TA has been exploited as an efficient hydrogen-bond donor for both polymersomes and PVPON at physiological $\mathrm{pH}$, producing multicomponent layer-by-layer capsules. ${ }^{7}$ These capsules were able to load plasmid DNA in the polymersome subcompartments, with triggered release in response to changes in $\mathrm{pH}$ between physiological and endocytic conditions. Moreover, the synthetic carriers provide possibilities for the incorporation of different functionalities and properties, allowing tuning of the therapeutic delivery outcome. TA can also form hydrogen bonds with the PEG chains in poloxamer 188 (F-68), resulting in self-assembled polyphenol-polymer nanoparticles (PPNPs) potentially useful for oral drug delivery for inflammatory bowel therapy (Figure 3a). ${ }^{30}$ These particles were loaded with the anti-inflammatory corticosteroid drug 
dexamethasone (DEX). The final complexed particles had a uniform spherical shape with a diameter of $\sim 60 \mathrm{~nm}$ and displayed targeting properties toward highly inflamed colons in mice owing to the electrostatic attraction between positively charged proteins in the inflamed colon epithelium and the negatively charged phenolic residues of the particles (Figure 3b). As polyphenols were hydrolyzed by esterase, approximately $62 \%$ of DEX was released after $4 \mathrm{~h}$ in a simulated colitis environment in the presence of $30 \mathrm{U} / \mathrm{mL}$ esterase.

More recently, the concept of "TANNylated proteins" was introduced, where proteins are functionalized with TA through hydrogen bonding. ${ }^{31}$ TANNylated green fluorescent protein (GFP) was prepared by mixing GFP with TA in solution (Figure 3c). The TANNylated GFP was stable at $\mathrm{pH} 7.4$ but aggregated when the solution $\mathrm{pH}$ decreased to 5.2 owing to enhanced hydrogen bonding arising from the protonated phenolic residues (Figure 3d). The TANNylated proteins showed prolonged blood circulation in rats, similar to the "stealth effect" induced by PEGylation. ${ }^{32}$ The TANNylated proteins penetrated the endothelium of blood vessels and accumulated in the myocardium of the heart owing to their strong affinity to proline-rich collagen and elastin in the heart rather than the glycocalyx in blood vessels. Notably, this TANNylation strategy can be applied to other components, such as peptides and viruses, for heart-targeting therapies. ${ }^{31}$

We recently reported a study on protein-polyphenol particles/capsules using TA and various proteins with different isoelectric point (pI), molecular weight $\left(M_{\mathrm{w}}\right)$, and aliphatic index. ${ }^{19}$ This allowed for a systematic investigation of the influence of the physicochemical properties on the formation of the protein-polyphenol assemblies. We hypothesized and demonstrated that the functional side chains of the different amino acids and the peptide backbone displayed multiple 
interactions with polyphenols, involving hydrophobic interactions, hydrogen bonding, and electrostatic interactions (Figure 3e). Five model proteins were used, i.e., lysozyme (LYZ), immunoglobulin $\mathrm{G}(\mathrm{IgG})$, hemoglobin ( $\mathrm{Hgb})$, glucose oxidase (GOx), and cytochrome C (CYC), to determine their interactions with polyphenols (Figure 3f). Urea degraded the IgG-TA and GOxTA capsules within $2 \mathrm{~h}$, suggesting that these two types of polyphenol-mediated protein assemblies were mainly stabilized by hydrogen bonds. The results also indicated that the strength of hydrogen bonding between the polyphenols and proteins increased with the higher $M_{\mathrm{w}}$ of the proteins as the peptide backbone and most of the exposed protein surface (polar amino acids) are hydrophilic. The remaining types of protein-polyphenol capsules did not disassemble in urea, suggesting that interactions other than hydrogen bonding are important is forming the protein-polyphenol assemblies. 


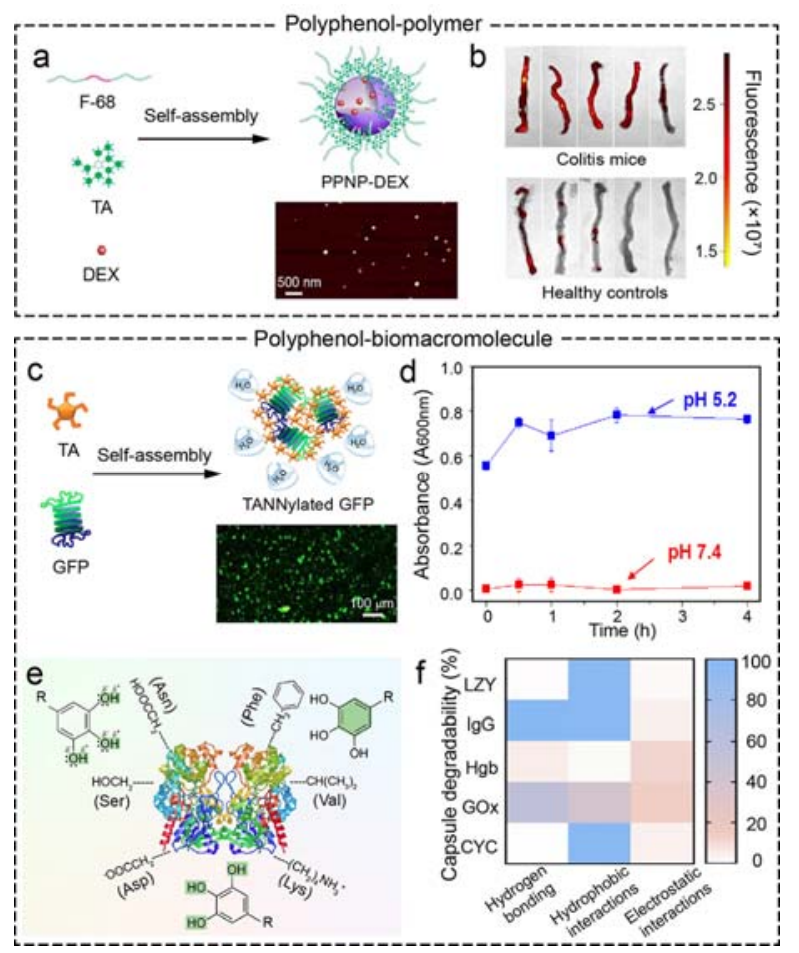

Figure 3. (a) Synthesis and characterization of PPNP-DEX nanoparticles by atomic force microscopy (AFM). (b) In vivo inflammation targeting in distal colons separated from colitis mice and healthy controls. The increased fluorescence intensity in colitis mice (relative to that displayed by healthy mice) demonstrated the targeting properties of PPNP-DEX toward inflamed colons. (c) Synthesis and fluorescence images of TANNylated GFP. (d) The stability of TANNylated GFP at $\mathrm{pH} 7.4$ and 5.2. The high absorbance of TANNylated GFP at $\mathrm{pH} 5.2$ indicates their aggregation due to enhanced hydrogen bonding. (e) Schematic diagram of the possible interactions between the functional groups of polyphenols and proteins. Ser: serine; Asn: asparagine; Phe: phenylalanine; Val: valine; Asp: aspartic acid; Lys: lysine. (f) Percentage of protein-TA capsules degraded after $1 \mathrm{~h}$ incubation in $100 \mathrm{mM}$ of urea, Tween 20 , or $\mathrm{NaCl}$, elucidating the different dominant interactions (i.e., hydrogen bonding, hydrophobic interactions, and electrostatic interactions). (a, b) Adapted with permission from ref. 30. Copyright 2018 American Chemical 
Society. (c, d) Adapted with permission from ref. 31. Copyright 2018 Springer Nature. (e, f) Adapted with permission from ref. 19. Copyright 2020 Wiley-VCH.

\section{2. $\pi$ Interactions}

Polyphenols are aromatic compounds with hydroxyl moieties and therefore have an electronrich $\pi$ system. Most reported $\pi$ interactions with polyphenols focus on aromatic $\pi-\pi$ stacking. ${ }^{12}$ Such stacking, with its confined steric structure, is typically stronger than van der Waals interactions formed between alkyl chains and provides efficient and predictable driving forces to assemble stable supramolecular structures. ${ }^{33}$ However, it is important to note that $\pi$ interactions are not restricted to this single class of interaction. Ethylene, acetylene, and other simple $\pi$ systems, as well as cations, also exhibit $\pi$ interactions with some polyphenol systems, although these cases are less studied.

Among the different materials that exhibit $\pi-\pi$ interactions, carbon materials, such as carbon nanotubes (CNTs) and graphene, are commonly combined with polyphenols. Using CNTs as a model, the adsorption of three aromatic compounds (i.e., salicylic acid, phthalic acid, and catechol) suggests that the main interaction between polyphenols and CNTs originates from $\pi-\pi$ interactions rather than hydrophobic interactions or electrostatic interactions. ${ }^{34}$ Furthermore, the adsorption of phenolic compounds on CNTs can be improved by using aromatic rings with a higher number of $-\mathrm{OH}$ group substituents. This indicates that electron-donating substituents strengthen the $\pi-\pi$ electron donor-acceptor interaction. ${ }^{35}$ Although not strictly particle assembly, by exploiting such strong $\pi-\pi$ interactions, TA can be used as a capping agent to modify the surface of graphene 
oxides and subsequently mediate their assembly into porous graphene oxides hydrogel systems that exhibited enhanced adsorption toward organic dyes and oils in water. ${ }^{36}$

Aromatic polymers, such as polystyrene (PS) particles, also interact with the aromatic rings of polyphenols. ${ }^{12}$ Molecular dynamic simulations demonstrated that the catechol and gallol groups of TA could interact with the aromatic styrene rings to anchor polyphenol molecules to the PS surface. TA by itself would interact too strongly with the PS surface to allow for secondary interactions; in contrast, $\mathrm{TA}_{3} / \mathrm{Fe}^{\mathrm{III}}$ complexes could interact with PS and extend into the solvent for secondary interactions (Figure $4 \mathrm{a}$ and $4 \mathrm{~b}$ ). Such interactions influenced the mobility of the polyphenol molecules once "locked" to the surface and were used to form complex hierarchical assemblies of particles for a variety of applications, including self-reporting drug release and magnetically directed bacteria. ${ }^{12}$

Drug-loaded micellar nanocomplexes (MNCs) can be formed from the anticancer drug doxorubicin (DOX) and the polyphenol-inspired molecule PEG-EGCG (Figure 4c). ${ }^{37}$ The strong $\pi-\pi$ stacking between the aromatic rings of both DOX and PEG-EGCG increased the solubility of DOX in aqueous solutions. A high DOX loading efficiency ( $~ 88 \%)$ was achieved by adjusting the PEG-EGCG-to-DOX weight ratio to 2. Importantly, the DOX-MNCs remained stable even after 100-fold dilution or incubation with phosphate-buffered saline (PBS) solution (Figure 4d) and could therefore provide a sustained release of DOX for 14 days. Compared with free DOX, the DOX-MNCs ( $\sim 124 \mathrm{~nm}$ in diameter) showed over 16-fold prolonged blood circulation and 20-fold greater selective accumulation in the tumor tissue at $24 \mathrm{~h}$ - these improvements were attributed to the enhanced permeability and retention effect (Figure 4e). This strategy illustrates a useful and 
relatively simple way to design functional supramolecular materials for sustained release using $\pi$ interactions.

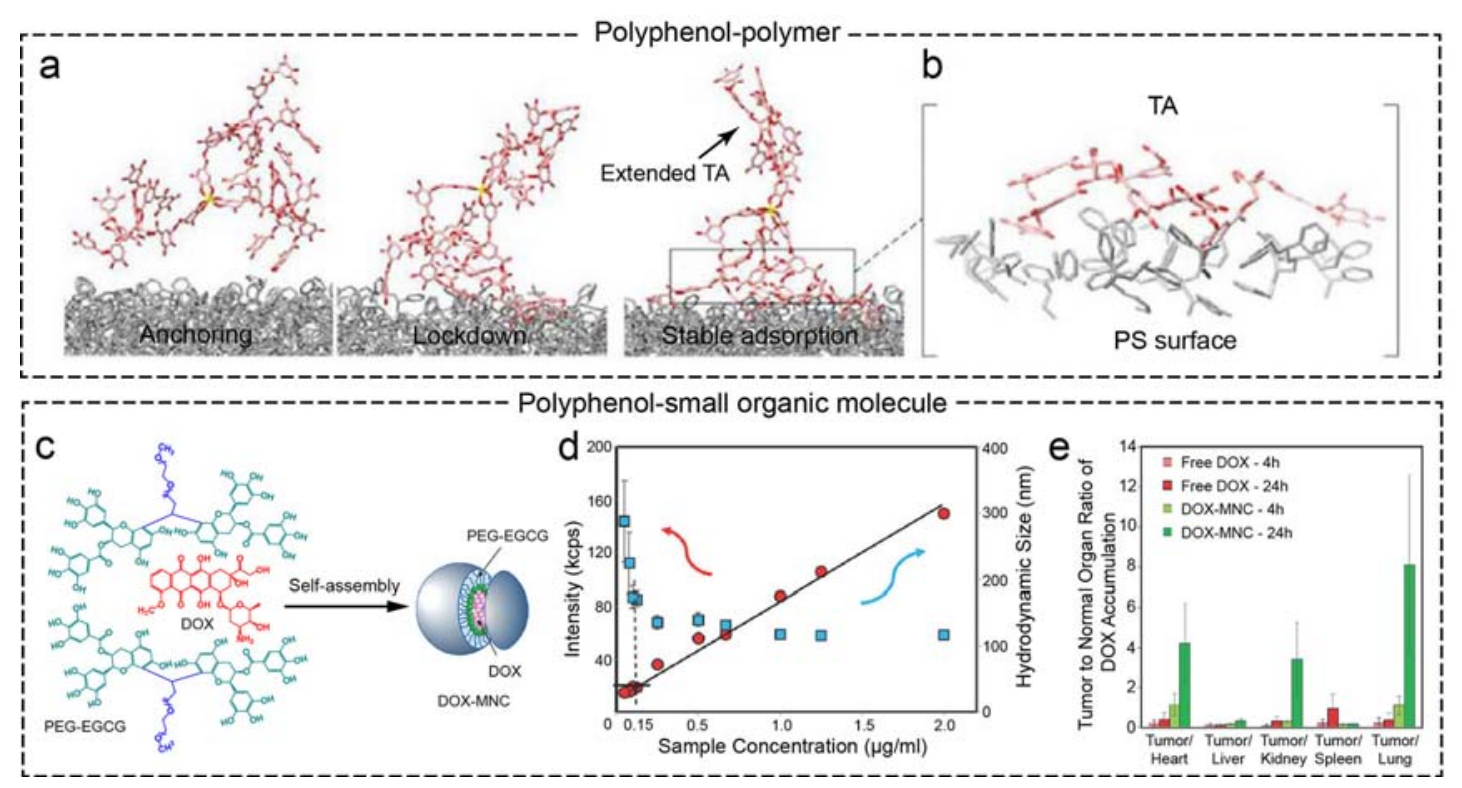

Figure 4. (a) Representative snapshots obtained from molecular dynamic simulations, showing the anchoring, lockdown, and stable adsorption steps between gallol groups of $\mathrm{TA} / \mathrm{Fe}^{\mathrm{III}}$ and a PS surface. One TA of $\mathrm{TA}_{3} / \mathrm{Fe}^{\mathrm{III}}$ remained unadsorbed on the PS surface and extended up to $4.5 \mathrm{~nm}$ away from the PS interface. This extended TA domain could provide an anchoring point for secondary interactions. (b) Close-up view of the aromatic interactions at the interface between TA and PS surface. (c) Schematic synthesis of DOX-MNCs. (d) Critical micelle concentration of DOX-MNCs determined by dynamic light scattering. (e) Tumor-to-organ DOX accumulation at 4 and $24 \mathrm{~h}$ post-administration of free DOX or DOX-MNCs, demonstrating the good tumor targeting of DOX-MNCs. $(a, b)$ Reproduced with permission from ref. 12. Copyright 2016 Springer Nature. (c-e) Reproduced with permission from ref. 37. Copyright 2018 Wiley-VCH. 
$\pi$ Interactions also play a role in the formation of polyphenol-protein complexes, especially for proteins containing aromatic amino acids and proline (e.g. thrombin, elastin, and collagen). ${ }^{31,38}$ Moreover, polyphenols can serve as Alzheimer's disease inhibitors possibly due to aromatic $\pi-\pi$ interactions with amyloidogenic proteins. ${ }^{39}$ Recently, the short-range cation- $\pi$ interaction between the trimethylammonium group on polyelectrolytes and the aromatic region of phenolic groups has been reported, ${ }^{40}$ and it is likely that $\pi$ interactions other than $\pi-\pi$ stacking will gain interest for both fundamental studies and material synthesis.

\subsection{Hydrophobic Interactions}

Hydrophobic interactions are an important driving force for protein folding and complexation in nature, and are widely recognized as the dominant mode of interaction between most proteins and polyphenols. ${ }^{41}$ For instance, to identify the major driving force for anticancer polyphenol-protein nanocomplexes self-assembled from EGCG and Herceptin, the influence of different competing interactions was investigated (Figure 5a). ${ }^{42}$ The micellar nanocomplexes were disassembled by Tween 20, Triton X-100, and sodium dodecyl sulphate (hydrophobic competitors), whereas urea (hydrogen bond competitor) and $\mathrm{NaCl}$ (ionic competitor) were ineffective in disrupting them. These results suggest that the dominant force driving self-assembly and stability in those complexes is hydrophobic. Similarly, we showed that CYC-TA, LYZ-TA, and IgG-TA capsules effectively disassembled in Tween 20 solution (Figure 5b), whereas a majority of all three capsule types remained intact after incubation with urea. ${ }^{19}$ In contrast, $50 \%$ and $75 \%$ of the GOx-TA and Hgb-TA capsules, respectively, remained intact after incubation with Tween 20, indicating different or additional intermolecular forces operating in these systems. Overall, we found that 
proteins with high aliphatic indexes (i.e., containing more aliphatic and hydrophobic side chains) formed stronger hydrophobic interactions with the aromatic groups of polyphenols compared with those with low aliphatic indexes (Figure 5b), although it is difficult to clearly delineate between hydrophobic and $\pi$ interactions. These results highlight the complex nature of polyphenol-protein interactions at the molecular level, which can be useful for constructing functional materials with other biomacromolecules.

To highlight the dominance of hydrophobic interactions between aromatic groups in polyphenols and hydrophobic surfaces, the adhesion properties of catechol-functionalized peptides on alkylterminated surfaces were examined. ${ }^{43}$ Along with simulations, these experimental results suggested that the catechol residues present in the peptides tend to adopt a parallel orientation $\left(<45^{\circ}\right.$ relative to the plane of the surface) near hydrophobic $\left(\mathrm{CH}_{3}\right.$-terminated) surfaces and a perpendicular orientation $\left(>45^{\circ}\right.$ relative to the plane of the surface) near hydrophilic $(\mathrm{OH}-$ terminated) surfaces. These observations indicate that polyphenols change their orientation to interact with different surfaces and may help explain why polyphenols are highly adherent.

Hydrophobic interactions also exist between the hydrophobic aromatic structures in polyphenols and various inorganic materials. For example, polyphenols were intercalated into transition metal dichalcogenide (TMD) (i.e., $\mathrm{MoS}_{2}$ ) nanosheets owing to the hydrophobic interaction between the $\mathrm{MoS}_{2}$ monolayer and the aromatic structures in polyphenols (Figure 5c). ${ }^{44}$ Additionally, the hydrophilic hydroxyl motifs of the polyphenols facilitated the colloidal stability of exfoliated nanosheets in aqueous solution. This demonstrates the synergistic effects of polyphenols for both assembly and colloidal stability (Figure 5d). 

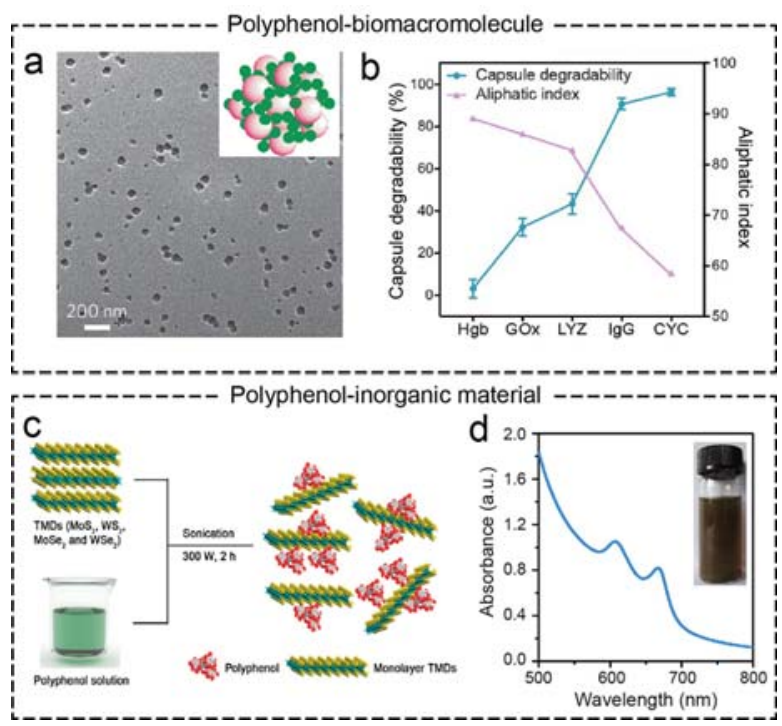

Figure 5. (a) Transmission electron microscopy image and schematic (inset) of the EGCG-protein complexes. (b) Relationship between the stability of protein-TA complexes and the aliphatic index of the proteins, as analyzed by capsule degradability in $100 \mathrm{mM}$ Tween 20. (c) Schematic of the polyphenol-assisted aqueous exfoliation process of TMD under sonication, which produces monolayer nanosheets. (d) UV-vis absorbance spectrum and photograph of the exfoliated $\mathrm{MoS}_{2}$ nanosheets after sonication in epigallocatechin solution. (a) Adapted with permission from ref. 42. Copyright 2014 Springer Nature. (b) Reproduced with permission from ref. 19. Copyright 2020 Wiley-VCH. (c, d) Reproduced with permission from ref. 44. Copyright 2018 American Chemical Society.

\subsection{Metal Coordination}

Polyphenols are well known to coordinate to various metal ions, including $\mathrm{Cu}^{2+}, \mathrm{Zn}^{2+}, \mathrm{Fe}^{2+}, \mathrm{Al}^{3+}$, $\mathrm{Fe}^{3+}, \mathrm{Zr}^{4+}$, and $\mathrm{Ti}^{4+}$, which is important in nature for plant pigmentation and cationic nutrient cycling. ${ }^{9,45}$ These complexes have different stoichiometries (e.g., mono-, bis-, tris-complex), as 
modulated by $\mathrm{pH}$, metal ion valency, or metal ion-to-phenolic group molar ratio. We previously described a rapid and universal method for coating various substrates through the coordination of polyphenols and metal ions, referred to as metal-phenolic networks (MPNs) (Figure 6a). ${ }^{8,46}$ This strategy is applicable to form core-shell particles, hollow capsules, and hierarchical superstructures with diverse surface chemistries, mechanical, magnetic, and electronic properties. ${ }^{12}$ MPNs disassemble at low $\mathrm{pH}$ upon protonation of the phenolic groups and disruption of the coordination bonds (Figure 6b), where tunable disassembly profiles can be generated from different metal ions. ${ }^{9}$ Moreover, the metal ions contribute additional functionality to the MPNs. For example, $\mathrm{Rh}^{3+}$-TA capsules displayed excellent catalytic properties for the hydrogenation of quinoline and $\mathrm{Fe}^{3+}$-TA systems acted as a magnetic resonance imaging probe. ${ }^{9,47}$ Recently, the coordination bonds of MPNs have been reported to facilitate the endosomal escape of nanoparticles as a result of the "proton-sponge effect", thereby enabling an alternative strategy for efficient intracellular drug delivery (Figure 6c). ${ }^{16}$ A multimodal theranostic polymeric nanovesicle (PNV) made of $\mathrm{Fe}^{3+} / \mathrm{Mn}^{2+}$-based MPNs (PNV@ $\mathrm{Fe}^{\mathrm{III}} \mathrm{Mn}^{\mathrm{II}} \mathrm{TA}$ ) was also designed, providing tumorspecific therapeutic regimens, including photoacoustic imaging, magnetic resonance imaging, and photothermal imaging and therapy (Figure 6d). ${ }^{48}$ In addition, our recent study has shown that MPN particles are highly biocompatible and biodegradable, as assessed following intratracheal administration in mice, with tunable aerodynamic properties for controlled pulmonary delivery. ${ }^{49}$ Polyphenols can directly interact with metal oxides (e.g. $\mathrm{TiO}_{2}, \mathrm{Fe}_{2} \mathrm{O}_{3}$, and $\mathrm{ZnO}$ ), where the two adjacent phenolic groups of catechols form bidentate mononuclear chelation complexes and/or bidentate binuclear bridging complexes with the metal oxides. ${ }^{50}$ The evolution of the catechol 
binding modes on $\mathrm{TiO}_{2}$ was studied, ${ }^{51}$ which indicated that after initial adsorption via $\mathrm{Ti}^{\mathrm{IV}}-$ catechol coordination, the hydration layer of the metal was disrupted and hydrogen bonds then formed between the metal surface and subsequent catechol groups (Figure 6e). When the surface sites became saturated, long-range electrostatic interactions could also occur. Binding between gallol moieties and $\mathrm{TiO}_{2}$ occurs in a similar way but the third phenolic hydroxyl group remains unbound. $^{52}$

It is notable that polyphenols are able to reduce noble metal ions, such as $\mathrm{Au}^{3+}, \mathrm{Pd}^{2+}$, and $\mathrm{Ag}^{+}$, to metal nanoclusters under suitable conditions and subsequently stabilize the nanoclusters through binding to the metal atoms on the surface..$^{53,54}$ The polyphenol-mediated synthesis of metal nanoparticles represents a versatile and green strategy compared with traditional wet chemical synthesis involving both reductants and surface capping agents. For example, monodisperse $\mathrm{Au}$ nanoparticles $(\sim 35 \mathrm{~nm})$ with an MPN shell were synthesized by mixing TA with $\mathrm{HAuCl}_{4}$ at room temperature. ${ }^{55}$ The presence of an MPN shell on the Au nanoparticles could modulate their catalytic performance, as the coating concentrated reactants (e.g., 4-nitrophenol) around the catalytic Au nanoparticles via $\pi-\pi$ stacking. This strategy may provide a new approach to modulating catalytic activity, with a view toward developing tailorable smart catalytic systems.

We recently developed a protocol for visualizing latent fingerprints (i.e., invisible residual biomolecule patterns) through the formation of $\mathrm{Ag}$ nanoparticles on pre-formed MPN films patterned by biomolecules (Figure 6f) ${ }^{56}$ Four major classes of biomolecules (proteins, lipids, nucleic acids, and polysaccharides) could interact with the MPN via various complex interactions. The deposited MPN films subsequently enabled the in situ reduction of $\mathrm{Ag}$ ions into $\mathrm{Ag}$ 
nanoparticles, resulting in high-fidelity patterns with enhanced reflectance and fluorescence. The potential to integrate multiple physicochemical properties could further investigation into polyphenol-mediated assembly methods and applications.

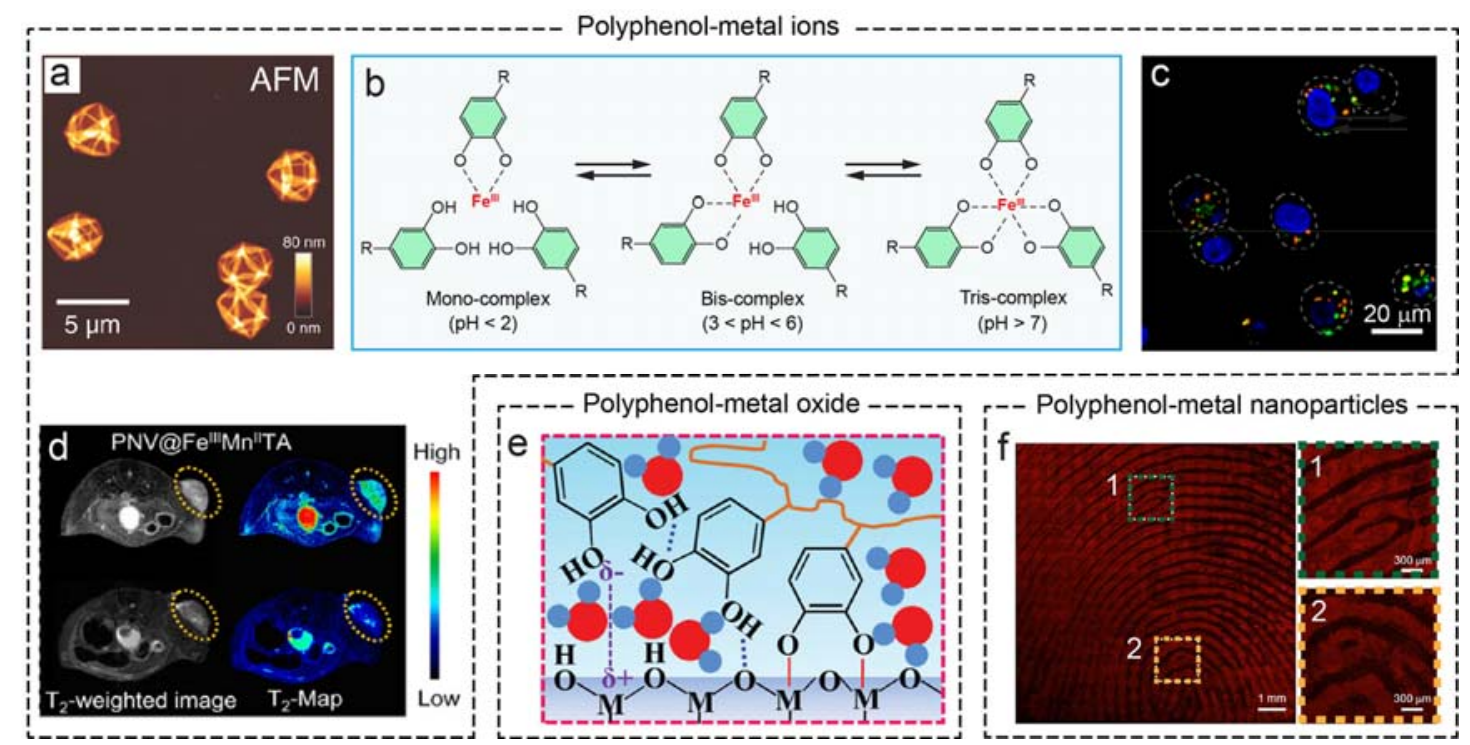

Figure 6. (a) AFM image of $\mathrm{Fe}^{\mathrm{III}}$-TA MPN capsules. (b) $\mathrm{pH}$-Dependent transition of $\mathrm{Fe}^{\mathrm{III}}$-catechol complexation state. $\mathrm{R}$ represents the remainder of the polyphenol molecule. (c) Representative fluorescence image of MDA-MB-231 cells incubated with silica nanoparticles (green) coated by Fe $\mathrm{III}^{\mathrm{III}} \mathrm{TA}$ for 4 h. Endo/lysosomes (red, LysoTracker Red), nuclei (blue, Hoechst 33342), and dashed lines indicate cellular boundaries. The low degree of colocalization of the green and red fluorescence signals suggested successful endosomal escape of the silica nanoparticles. (d) Representative in vivo T2-weighted MRI scans and pseudocolor maps of 4T1-tumor-bearing mice before and $4 \mathrm{~h}$ after intravenous injection of PNV@Fe ${ }^{\mathrm{III}} \mathrm{Mn}^{\mathrm{II}} \mathrm{TA}$. Tumors are marked with yellow circles. (e) Schematic of the adsorption mechanism of catechol groups on a wet metal hydroxide surface. (f) Confocal fluorescence images of $\mathrm{Ag}$ nanoparticles on an MPN-coated fingerprint excited at $561 \mathrm{~nm}$. (a, b) Reproduced with permission from ref. 8. Copyright 2013 American 
Association for the Advancement of Science. (c) Reproduced with permission from ref. 16. Copyright 2019 American Chemical Society. (d) Reproduced with permission from ref. 48. Copyright 2018 American Chemical Society. (e) Reproduced with permission from ref. 51. Copyright 2016 Wiley-VCH. (f) Reproduced with permission from ref. 56. Copyright 2020 Wiley$\mathrm{VCH}$.

\subsection{Covalent Bonds}

Polyphenols commonly undergo oxidative coupling induced by oxidants, enzymes, or increases in $\mathrm{pH}$ or temperature to form oligo/polymeric polyphenolic assemblies. ${ }^{57}$ For example, the catechol and pyrogallol groups of EGCG can be converted into corresponding highly reactive semiquinones or quinones upon the addition of $\mathrm{Cu}^{2+}$ at elevated temperatures. The coupling formation of interflavan-linked dehydrodicatechins $\left(\mathrm{T}-\mathrm{T}^{\prime}\right)$ then occurs through nucleophilic addition by a quinone and/or coupling reactions by a semiquinone radical (Figure $7 \mathrm{a}$ ). ${ }^{58}$ These polymeric polyphenols then assembled into microspheres with a diameter of $1-3 \mu \mathrm{m}$ and exhibited green fluorescence under UV irradiation (Figure $7 \mathrm{~b}$ and $7 \mathrm{c}$ ). Recently, the polymerized polyphenol-based particles from quercetin were used to load hydrophobic drugs (e.g., curcumin) in a one-pot method. ${ }^{59}$

Furthermore, polyphenols react with both small molecules and macromolecules. One welldocumented example is that aldehydes cross-link polyphenols in the presence of acetic acid or hydrochloric acid, ${ }^{60,61}$ wherein the protonation of acetaldehyde results in a carbocation that attacks the aromatic rings of the polyphenol, followed by nucleophilic attack by another polyphenol. 
Notably, stabilizing polyphenol-based materials with such covalent bonds considerably increases the stability of the assemblies, which may be beneficial for environmental applications such as metal ion sequestration. ${ }^{62}$ In addition, the phenolic groups in polyphenols can be used as anchors for diverse functional groups such as epoxy groups ${ }^{63}$ and alkyl bromides. ${ }^{64}$ Similarly, quinones can react with nucleophilic functional groups (e.g. $-\mathrm{NH}_{2}$ and $-\mathrm{SH}$ ) by Michael addition and/or Schiff-base reaction, providing additional covalent modification routes for polyphenols. Furthermore, the presence of some specific functional groups, such as carboxyl groups, in certain polyphenols (e.g. caffeic acid) offers additional possibilities for tandem conjugation. ${ }^{65}$ Taking advantage of the dynamic nature of boronate ester chemistry, wherein a reversible covalent bond is formed between boronic acid and the cis-diol in some polyphenols, a responsive boronatephenolic network was developed. These complexes were stable at alkaline $\mathrm{pH}$ but disassemble at acidic $\mathrm{pH}$ and/or in the presence of exogenous competing cis-diols (e.g., mannitol) (Figure 7d and 7e), holding great promise for biomedicine. ${ }^{10,47}$ The extensive chemical reactivity of polyphenols has been recently summarized elsewhere. ${ }^{65}$ 


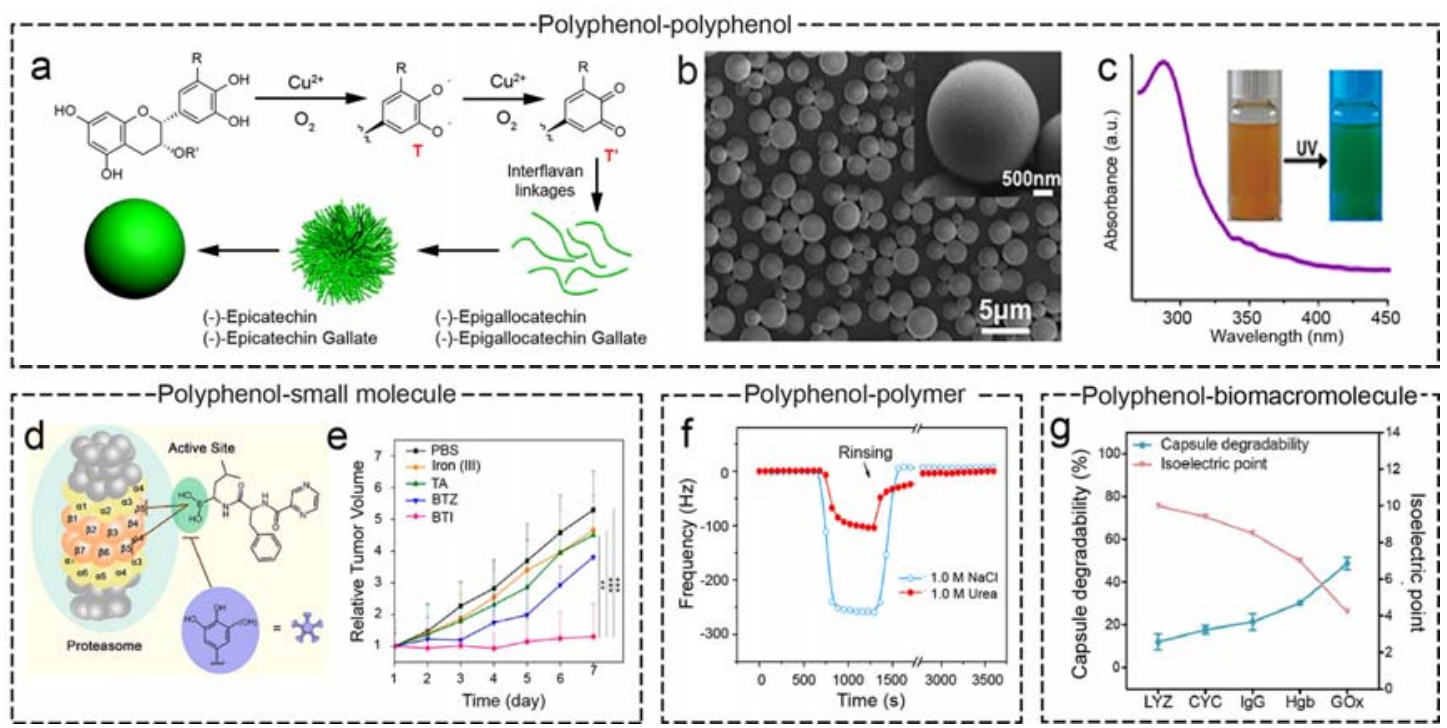

Figure 7. (a) Schematic illustration of $\mathrm{Cu}^{\mathrm{II}}$-mediated oxidative assembly of tea polyphenols at elevated temperature $\left(100{ }^{\circ} \mathrm{C}\right)$. (b) Scanning electron microscopy image of the resulting polymerized particles. (c) UV-vis spectrum of the particle solution. Inset: photograph showing the green fluorescence of the solution under UV irradiation. (d) Schematic showing the competing molecular interactions among bortezomib (BTZ), polyphenols and proteasome following intracellular delivery of boronate-phenolic complexes. (e) Relative tumor volume in MDA-MB231 tumor-bearing mice after intravenous administration of different materials. The decreased tumor volume observed following treatment with BTI (i.e., BTZ-TA-Fe ${ }^{3+}$ ) indicated their improved therapeutic efficacy over other materials examined. (f) Stability of TA/poly(sulfobetaine methacrylate) (PSBMA) film in $\mathrm{NaCl}$ and urea solutions, as analyzed by quartz crystal microgravimetry. (g) Relationship between the stability of protein-TA complexes and isoelectric point of the proteins, as analyzed by capsule degradability in $100 \mathrm{mM} \mathrm{NaCl}$. (a-c) Adapted with permission from ref. 58. Copyright 2013 American Chemical Society. (d, e) Adapted with permission from ref. 47. Copyright 2018 American Chemical Society. (f) Reproduced with 
permission from ref. 66. Copyright 2015 American Chemical Society. (g) Reproduced with permission from ref. 19. Copyright 2020 Wiley-VCH.

\subsection{Electrostatic Interactions}

Electrostatic interactions generate ion-paring complexes, which are widely used for biological recognition and macromolecular configuration stabilization. Polyphenols become negatively charged at higher $\mathrm{pH}$ owing to the ionization of the phenolic groups above their $\mathrm{p} K_{\mathrm{a}}$, giving rise to potential electrostatic interactions. Such interactions are responsive to polyelectrolyte concentration and surrounding conditions, including $\mathrm{pH}$, ionic strength, and temperature, all of which can be modulated for specific applications.

In the system of multilayers of TA/PSBMA, the phenolic groups of TA ( $\left.\mathrm{p} K_{\mathrm{a}} 8.5\right)$ were deprotonated and negatively charged at $\mathrm{pH} 10$ and could therefore interact with the positively charged quaternary ammonium group of PSBMA. ${ }^{66}$ In contrast, the multilayers disassembled at $\mathrm{pH} 3$, suggesting that protonation of the phenolic groups and the neutral state of TA disrupt the electrostatic interactions. Moreover, an increase in the ionic strength could disassemble the multilayers by disturbing the electrostatic bonds (Figure 7f). Although this work described film formation on planar substrates, it highlights the characteristic electrostatic properties of TA in a dynamic way, which also provides useful information for assembling functional and dynamic particle systems.

Our recent protein-polyphenol study demonstrated that LYZ-TA capsules disassembled most efficiently in $100 \mathrm{mM} \mathrm{NaCl}$ compared to other protein-polyphenol capsules as a result of ionic 
shielding (Figure 7g). ${ }^{19}$ As the $\mathrm{pI}$ of the proteins increases in the order of $\mathrm{GOx}<\mathrm{Hgb}<\mathrm{IgG}<$ CYC $<$ LYZ, side chains in the protein became more positively charged and thus enhanced ionic interactions with the deprotonated hydroxyl groups of the catechol/gallol; thus electrostatic interactions were likely the dominant interactions in the assembly of LYZ-TA. Moreover, there is a large body of research on the electrostatic interactions between polyphenols and positively charged molecules, such as amine-containing molecules, although contribution from hydrogen bonding cannot be discarded. For example, mesoporous silica nanoparticles (MSNs) could be templated by supramolecular complexes of TA and ammonium cations. ${ }^{67}$ The produced supramolecular complexes could subsequently interact with silicate species, promoting the formation of hybrid nanoparticles. MSNs were obtained after removing the complexes simply by washing with water. By adjusting the concentration of TA, the pore size was tuned from 5.9 to $12.9 \mathrm{~nm}$. These MSNs showed the capacity to immobilize and protect bioactive macromolecules (e.g., enzymes), with implications for the controlled release of proteins.

\section{OUTLOOK}

In this Account, we focused on the various physicochemical interactions that arise between polyphenols and different materials and how those interactions govern the assembly, disassembly, and functionality of the particles depending on the desired application. Although this Account provides a succinct overview of the interactions relevant for the design of polyphenol-based particles, a better understanding of the interaction chemistries ranging from the nanoscale to the molecular scale is still needed. The complex nature of polyphenols means that it is unlikely for a system to solely contain a single type of interaction even though studies generally focus on the 
dominant interaction. For example, this could indicate that chelation-based systems, such as MPNs, might also be stabilized through hydrogen bonding, hydrophobic or $\pi$ interactions arising between the polyphenols themselves.

In addition, there is much scope for both fundamental and applied research in this field, as various gaps currently exist in the literature. We herein highlight some possible areas of research to help further advance the field. (1) Elucidating how the interplay of dominant and non-dominant interactions alter the stability and responsiveness of polyphenol-based particles is an essential fundamental question to address, which may be further strengthened by simulation studies. (2) Similarly, investigating changes in the interaction type and strength in different environments will shed light on the assembly/disassembly mechanisms of particles and potentially open up further applications. Understanding the dynamic nature of interactions under different conditions should also lead to more concrete guidelines for synthesizing responsive particles for applications in complex environments. (3) Exploring how multiple interaction pathways can be controlled through careful choice of precursors is important for further rational design. (4) Broadening the range of materials (by examining blends of multiple materials) assembled with polyphenols has the potential to lead to synergistic or emergent properties. (5) Expanding the choice of phenolics offers a wide range of possibilities for controlling synthesis, as most polyphenol-inspired particles reported to date are based on a handful of candidates (e.g. TA, EGCG, PEG-catechol). Functional polyphenols, for example therapeutic polyphenol species, could expand the application of polyphenol-inspired particles, especially for nanomedicine. (6) Establishing scalable methods for synthesizing and extracting polyphenols with defined chemical structures is essential for further 
particle development. As many polyphenols are inexpensive and naturally abundant, their commercial implementation is both feasible and potentially lucrative. (7) Finally, exploring the multifaceted chemistry and distinct structure of polyphenols to enable the complex manipulation of particle surface chemistry should offer opportunities not readily accessible for conventional colloidal systems. Although much has been done to assemble and apply polyphenol-based materials, there are still significant advances to be achieved. We envision that, with the concerted efforts of researchers, understanding the role of diverse interactions involved in the formation of polyphenol assemblies will lead to increasingly sophisticated functional materials for various applications.

\section{AUTHOR INFORMATION}

\section{ORCID}

Jiajing Zhou: 0000-0001-5203-4737

Zhixing Lin: 0000-0001-9372-3424

Yi Ju: 0000-0003-0103-1207

Md. Arifur Rahim: 0000-0002-7994-2097

Joseph J. Richardson: 0000-0001-8618-4127

Frank Caruso: 0000-0002-0197-497X

\section{Notes}

The authors declare no competing financial interest.

\section{Biographies}


Jiajing Zhou completed his Ph.D. in 2016 at Nanyang Technological University. He has been working with Frank Caruso as a postdoctoral fellow at The University of Melbourne since 2018. His research focuses on the synthesis of functional nanohybrids for biomedical applications.

Zhixing Lin received his M.Eng. degree in Chemical Engineering from Shanghai Jiao Tong University in 2017. He is currently a Ph.D. student under the supervision of Frank Caruso at The University of Melbourne. His research focuses on functional materials for biotechnology.

Yi Ju completed his Ph.D. in 2016 under the supervision of Frank Caruso at The University of Melbourne and is currently a postdoctoral fellow in Frank Caruso's group. His research focuses on understanding how nanomaterials interact with complex biological systems.

Md. Arifur Rahim completed his Ph.D. in 2016 under the supervision of Frank Caruso at The University of Melbourne. Following his Ph.D completion, he undertook a postdoctoral fellow position in the same group exploring various metal-phenolic materials. Recently, he moved to the University of New South Wales as a postdoctoral fellow where he investigates the fundamentals of composite materials based on polyphenols and liquid metals.

Joseph J. Richardson completed his Ph.D. in 2015, researching thin-film deposition strategies under the supervision of Frank Caruso at The University of Melbourne. After a 2-year postdoctoral fellowship at CSIRO studying metal-organic hybrid systems, he returned to Frank Caruso's group to investigate the fundamentals governing metal-organic thin film assembly.

Frank Caruso received his Ph.D. in 1994 from The University of Melbourne and thereafter conducted postdoctoral research at the CSIRO Division of Chemicals and Polymers. He was an Alexander von Humboldt Research Fellow and a group leader at the Max Planck Institute of Colloids and Interfaces from 1997 to 2002. Since 2003, he has been a professor and is currently 
an NHMRC Senior Principal Research Fellow at The University of Melbourne. He is Deputy Director of the ARC Centre of Excellence in Convergent Bio-Nano Science and Technology. His research focuses on developing advanced nano- and biomaterials for biotechnology and medicine.

\section{ACKNOWLEDGMENTS}

This research was conducted and funded by the Australian Research Council (ARC) Centre of Excellence in Convergent Bio-Nano Science and Technology (project number CE140100036), the ARC through the Discovery Project Scheme (DP170103331), and a National Health and Medical Research Council (NHMRC) Project Grant (APP1138361). F.C. acknowledges the award of an NHMRC Senior Principal Research Fellowship (APP1135806). The authors acknowledge Dr. Gyeongwon Yun, Yiyuan Han, and Denzil Furtado for helpful discussions.

\section{REFERENCES}

(1) Bravo, L. Polyphenols: Chemistry, Dietary Sources, Metabolism, and Nutritional Significance. Nutr. Rev. 1998, 56, 317-333.

(2) Quideau, S.; Deffieux, D.; Douat-Casassus, C.; Pouysegu, L. Plant Polyphenols: Chemical Properties, Biological Activities, and Synthesis. Angew. Chem. Int. Ed. 2011, 50, 586-621.

(3) Haslam, E. Practical Polyphenolics: From Structure to Molecular Recognition and Physiological Action. Cambridge University Press: New York, 1998.

(4) Rahim, M. A.; Kristufek, S. L.; Pan, S.; Richardson, J. J.; Caruso, F. Phenolic Building Blocks for the Assembly of Functional Materials. Angew. Chem. Int. Ed. 2019, 58, 1904-1927.

(5) Shin, M.; Park, E.; Lee, H. Plant-Inspired Pyrogallol-Containing Functional Materials. $A d v$. Funct. Mater. 2019, 29, 1903022.

(6) Guo, J.; Suma, T.; Richardson, J. J.; Ejima, H. Modular Assembly of Biomaterials Using Polyphenols as Building Blocks. ACS Biomater. Sci. Eng. 2019, 5, 5578-5596.

(7) Lomas, H.; Johnston, A. P.; Such, G. K.; Zhu, Z.; Liang, K.; Van Koeverden, M. P.; Alongkornchotikul, S.; Caruso, F. Polymersome-Loaded Capsules for Controlled Release of DNA. Small 2011, 7, 2109-2119.

(8) Ejima, H.; Richardson, J. J.; Liang, K.; Best, J. P.; van Koeverden, M. P.; Such, G. K.; Cui, J.; Caruso, F. One-Step Assembly of Coordination Complexes for Versatile Film and Particle Engineering. Science 2013, 341, 154-157.

(9) Guo, J.; Ping, Y.; Ejima, H.; Alt, K.; Meissner, M.; Richardson, J. J.; Yan, Y.; Peter, K.; Von Elverfeldt, D.; Hagemeyer, C. E.; Caruso, F. Engineering Multifunctional Capsules through the Assembly of Metal-Phenolic Networks. Angew. Chem. Int. Ed. 2014, 53, 5546-5551.

(10)Guo, J.; Sun, H.; Alt, K.; Tardy, B. L.; Richardson, J. J.; Suma, T.; Ejima, H.; Cui, J.; 
Hagemeyer, C. E.; Caruso, F. Boronate-Phenolic Network Capsules with Dual Response to Acidic pH and cis-Diols. Adv. Healthcare Mater. 2015, 4, 1796-1801.

(11) Ju, Y.; Cui, J.; Müllner, M.; Suma, T.; Hu, M.; Caruso, F. Engineering Low-Fouling and pHDegradable Capsules through the Assembly of Metal-Phenolic Networks. Biomacromolecules 2015, 16, 807-814.

(12)Guo, J.; Tardy, B. L.; Christofferson, A. J.; Dai, Y.; Richardson, J. J.; Zhu, W.; Hu, M.; Ju, Y.; Cui, J.; Dagastine, R. R.; Yarovsky, I.; Caruso, F. Modular Assembly of Superstructures from Polyphenol-Functionalized Building Blocks. Nat. Nanotechnol. 2016, 11, 1105-1111.

(13) Ju, Y.; Dai, Q.; Cui, J.; Dai, Y.; Suma, T.; Richardson, J. J.; Caruso, F. Improving Targeting of Metal-Phenolic Capsules by the Presence of Protein Coronas. ACS Appl. Mater. Interfaces 2016, 8, 22914-22922.

(14) Dai, Y.; Guo, J.; Wang, T. Y.; Ju, Y.; Mitchell, A. J.; Bonnard, T.; Cui, J.; Richardson, J. J.; Hagemeyer, C. E.; Alt, K.; Caruso, F. Self-Assembled Nanoparticles from Phenolic Derivatives for Cancer Therapy. Adv. Healthcare Mater. 2017, 6, 1700467.

(15) Rahim, M. A.; Hata, Y.; Björnmalm, M.; Ju, Y.; Caruso, F. Supramolecular Metal-Phenolic Gels for the Crystallization of Active Pharmaceutical Ingredients. Small 2018, 14, 1801202.

(16)Chen, J.; Li, J.; Zhou, J.; Lin, Z.; Cavalieri, F.; Czuba-Wojnilowicz, E.; Hu, Y.; Glab, A.; Ju, Y.; Richardson, J. J.; Caruso, F. Metal-Phenolic Coatings as a Platform to Trigger Endosomal Escape of Nanoparticles. ACS Nano 2019, 13, 11653-11664.

(17)Pan, S.; Guo, R.; Bertleff-Zieschang, N.; Li, S.; Besford, Q. A.; Zhong, Q. Z.; Yun, G.; Zhang, Y.; Cavalieri, F.; Ju, Y.; Goudeli, E.; Richardson, J. J.; Caruso, F. Modular Assembly of Host-Guest Metal-Phenolic Networks Using Macrocyclic Building Blocks. Angew. Chem. Int. Ed. 2020, 59, 275-280.

(18)Lin, Z.; Zhou, J.; Cortez-Jugo, C.; Han, Y.; Ma, Y.; Pan, S.; Hanssen, E.; Richardson, J. J.; Caruso, F. Ordered Mesoporous Metal-Phenolic Network Particles. J. Am. Chem. Soc. 2020, 142, 335-341.

(19) Han, Y.; Lin, Z.; Zhou, J.; Yun, G.; Guo, R.; Richardson, J. J.; Caruso, F. Polyphenol-Mediated Assembly of Proteins for Engineering Functional Materials. Angew. Chem. Int. Ed. 2020, DOI: http://dx.doi.org/ DOI: 10.1002/ange.202002089.

(20) Sileika, T. S.; Barrett, D. G.; Zhang, R.; Lau, K. H.; Messersmith, P. B. Colorless Multifunctional Coatings Inspired by Polyphenols Found in Tea, Chocolate, and Wine. Angew. Chem. Int. Ed. 2013, 52, 10766-10770.

(21) Muzolf, M.; Szymusiak, H.; Gliszczyńska-Świgło, A.; Rietjens, I. M.; Tyrakowska, B. pHDependent Radical Scavenging Capacity of Green Tea Catechins. J. Agric. Food Chem. 2008, 56, 816-823.

(22) Rahim, M. A.; Ejima, H.; Cho, K. L.; Kempe, K.; Müllner, M.; Best, J. P.; Caruso, F. Coordination-Driven Multistep Assembly of Metal-Polyphenol Films and Capsules. Chem. Mater. 2014, 26, 1645-1653.

(23) Kim, B. S.; Lee, H. I.; Min, Y.; Poon, Z.; Hammond, P. T. Hydrogen-Bonded Multilayer of pH-Responsive Polymeric Micelles with Tannic Acid for Surface Drug Delivery. Chem. Commun. 2009, 28, 4194-4196. 
(24) Chen, J.; Kozlovskaya, V.; Goins, A.; Campos-Gomez, J.; Saeed, M.; Kharlampieva, E. Biocompatible Shaped Particles from Dried Multilayer Polymer Capsules. Biomacromolecules 2013, 14, 3830-3841.

(25) Shutava, T.; Prouty, M.; Kommireddy, D.; Lvov, Y. pH Responsive Decomposable Layer-byLayer Nanofilms and Capsules on the Basis of Tannic Acid. Macromolecules 2005, 38, 2850-2858. (26) Shin, M.; Ryu, J. H.; Park, J. P.; Kim, K.; Yang, J. W.; Lee, H. DNA/Tannic Acid Hybrid Gel Exhibiting Biodegradability, Extensibility, Tissue Adhesiveness, and Hemostatic Ability. Adv. Funct. Mater. 2015, 25, 1270-1278.

(27) Jakobek, L. Interactions of Polyphenols with Carbohydrates, Lipids and Proteins. Food Chem. 2015, 175, 556-567.

(28) Yu, J.; Wei, W.; Danner, E.; Israelachvili, J. N.; Waite, J. H. Effects of Interfacial Redox in Mussel Adhesive Protein Films on Mica. Adv. Mater. 2011, 23, 2362-2366.

(29) Amorati, R.; Valgimigli, L. Modulation of the Antioxidant Activity of Phenols by NonCovalent Interactions. Org. Biomol. Chem. 2012, 10, 4147-4158.

(30) Wang, X.; Yan, J.-J.; Wang, L.; Pan, D.; Yang, R.; Xu, Y.; Sheng, J.; Huang, Q.; Zhao, H.; Yang, M. Rational Design of Polyphenol-Poloxamer Nanovesicles for Targeting Inflammatory Bowel Disease Therapy. Chem. Mater. 2018, 30, 4073-4080.

(31) Shin, M.; Lee, H.-A.; Lee, M.; Shin, Y.; Song, J.-J.; Kang, S.-W.; Nam, D.-H.; Jeon, E. J.; Cho, M.; Do, M.; Park, S.; Lee, M. S.; Jang, J.-H.; Cho, S.-W.; Kim, K.-S.; Lee, H. Targeting Protein and Peptide Therapeutics to the Heart via Tannic Acid Modification. Nat. Biomed. Eng. 2018, 2, 304-317.

(32) Cui, J.; De Rose, R.; Alt, K.; Alcantara, S.; Paterson, B. M.; Liang, K.; Hu, M.; Richardson, J. J.; Yan, Y.; Jeffery, C. M.; Price, R. I.; Peter, K.; Hagemeyer, H. E.; Donnelly, P. S.; Kent, S. J.; Caruso, F. Engineering Poly(ethylene Glycol) Particles for Improved Biodistribution. ACS Nano 2015, 9, 1571-1580.

(33) Martinez, C. R; Iverson, B. L. Rethinking the Term "Pi-Stacking". Chem. Sci. 2012, 3, 21912201.

(34) Liu, F. F.; Fan, J. L.; Wang, S. G.; Ma, G. H. Adsorption of Natural Organic Matter Analogues by Multi-Walled Carbon Nanotubes: Comparison with Powdered Activated Carbon. Chem. Eng. J. 2013, 219, 450-458.

(35) Lin, D.; Xing, B. Tannic Acid Adsorption and Its Role for Stabilizing Carbon Nanotube Suspensions. Environ. Sci. Technol. 2008, 42, 5917-5923.

(36) Luo, J.; Lai, J.; Zhang, N.; Liu, Y.; Liu, R.; Liu, X. Tannic Acid Induced Self-Assembly of Three-Dimensional Graphene with Good Adsorption and Antibacterial Properties. ACS Sustainable Chem. Eng. 2016, 4, 1404-1413.

(37) Liang, K.; Chung, J. E.; Gao, S. J.; Yongvongsoontorn, N.; Kurisawa, M. Highly Augmented Drug Loading and Stability of Micellar Nanocomplexes Composed of Doxorubicin and Poly(ethylene Glycol)-Green Tea Catechin Conjugate for Cancer Therapy. Adv. Mater. 2018, 30, 1706963.

(38) Zondlo, N. J. Aromatic-Proline Interactions: Electronically Tunable $\mathrm{CH} / \pi$ Interactions. Acc. Chem. Res. 2012, 46, 1039-1049. 
(39) Engel, M. F.; vandenAkker, C. C.; Schleeger, M.; Velikov, K. P.; Koenderink, G. H.; Bonn, M. The Polyphenol EGCG Inhibits Amyloid Formation Less Efficiently at Phospholipid Interfaces Than in Bulk Solution. J. Am. Chem. Soc. 2012, 134, 14781-14788.

(40) Kim, S.; Huang, J.; Lee, Y.; Dutta, S.; Yoo, H. Y.; Jung, Y. M.; Jho, Y.; Zeng, H.; Hwang, D. S. Complexation and Coacervation of Like-Charged Polyelectrolytes Inspired by Mussels. Proc. Natl. Acad. Sci. U. S. A. 2016, 113, E847-E853.

(41) Oh, H. I.; Hoff, J. E.; Armstrong, G. S.; Haff, L. A. Hydrophobic Interaction in Tannin-Protein Complexes. J. Agric. Food Chem. 1980, 28, 394-398.

(42) Chung, J. E.; Tan, S.; Gao, S. J.; Yongvongsoontorn, N.; Kim, S. H.; Lee, J. H.; Choi, H. S.; Yano, H.; Zhuo, L.; Kurisawa, M.; Ying, J. Y. Self-Assembled Micellar Nanocomplexes Comprising Green Tea Catechin Derivatives and Protein Drugs for Cancer Therapy. Nat. Nanotechnol. 2014, 9, 907-912.

(43) Levine, Z. A.; Rapp, M. V.; Wei, W.; Mullen, R. G.; Wu, C.; Zerze, G. H.; Mittal, J.; Waite, J. H.; Israelachvili, J. N.; Shea, J. E. Surface Force Measurements and Simulations of MusselDerived Peptide Adhesives on Wet Organic Surfaces. Proc. Natl. Acad. Sci. U. S. A. 2016, 113, 4332-4337.

(44) Zhang, C.; Hu, D. F.; Xu, J. W.; Ma, M. Q.; Xing, H.; Yao, K.; Ji, J.; Xu, Z. K. PolyphenolAssisted Exfoliation of Transition Metal Dichalcogenides into Nanosheets as Photothermal Nanocarriers for Enhanced Antibiofilm Activity. ACS Nano 2018, 12, 12347-12356.

(45) Lattanzio, V.; Kroon, P. A.; Quideau, S.; Treutter, D. Plant Phenolics - Secondary Metabolites with Diverse Functions. Recent Adv. Polyphenol Res. 2008, 1, 1-35.

(46) Ejima, H.; Richardson, J. J.; Caruso, F. Metal-Phenolic Networks as a Versatile Platform to Engineer Nanomaterials and Biointerfaces. Nano Today 2017, 12, 136-148.

(47) Wang, C.; Sang, H.; Wang, Y.; Zhu, F.; Hu, X.; Wang, X.; Wang, X.; Li, Y.; Cheng, Y. Foe to Friend: Supramolecular Nanomedicines Consisting of Natural Polyphenols and Bortezomib. Nano Lett. 2018, 18, 7045-7051.

(48) Liu, T.; Zhang, M.; Liu, W.; Zeng, X.; Song, X.; Yang, X.; Zhang, X.; Feng, J. Metal Ion/Tannic Acid Assembly as a Versatile Photothermal Platform in Engineering Multimodal Nanotheranostics for Advanced Applications. ACS Nano 2018, 12, 3917-3927.

(49) Ju, Y.; Cortez-Jugo, C.; Chen, J.; Wang, T. Y.; Mitchell, A. J.; Tsantikos, E.; Bertleff-Zieschang, N.; Lin, Y. W.; Song, J.; Cheng, Y.; Mettu, S.; Rahim, M. A.; Pan, S.; Yun, G.; Hibbs, M. L.; Yeo, L. Y.; Hagemeyer, C. E.; Caruso, F. Engineering of Nebulized Metal-Phenolic Capsules for Controlled Pulmonary Deposition. Adv. Sci. 2020, 7, 1902650.

(50) Li, S. C.; Wang, J. G.; Jacobson, P.; Gong, X. Q.; Selloni, A.; Diebold, U. Correlation Between Bonding Geometry and Band Gap States at Organic-Inorganic Interfaces: Catechol on Rutile $\mathrm{TiO}_{2}$ (110). J. Am. Chem. Soc. 2009, 131, 980-984.

(51) Wei, W.; Petrone, L.; Tan, Y.; Cai, H.; Israelachvili, J. N.; Miserez, A.; Waite, J. H. An Underwater Surface-Drying Peptide Inspired by a Mussel Adhesive Protein. Adv. Funct. Mater. 2016, 26, 3496-3507.

(52) Janković, I. A.; Šaponjić, Z. V.; Džunuzović, E. S.; Nedeljković, J. M. New Hybrid Properties of $\mathrm{TiO}_{2}$ Nanoparticles Surface Modified with Catecholate Type Ligands. Nanoscale Res. Lett. 2010, 
$5,81-88$.

(53) Fei, J.; Zhao, J.; Du, C.; Wang, A.; Zhang, H.; Dai, L.; Li, J. One-Pot Ultrafast Self-Assembly of Autofluorescent Polyphenol-Based Core@Shell Nanostructures and Their Selective Antibacterial Applications. ACS Nano 2014, 8, 8529-8536.

(54) Nadagouda, M. N.; Varma, R. S. Green Synthesis of Silver and Palladium Nanoparticles at Room Temperature Using Coffee and Tea Extract. Green Chem. 2008, 10, 859-862.

(55) Zeng, T.; Zhang, X.; Guo, Y.; Niu, H.; Cai, Y. Enhanced Catalytic Application of $\mathrm{Au} @$ Polyphenol-Metal Nanocomposites Synthesized by a Facile and Green Method. J. Mater. Chem. A 2014, 2, 14807-14811.

(56)Yun, G.; Richardson, J. J.; Capelli, M.; Hu, Y.; Besford, Q. A.; Weiss, A. C.; Lee, H.; Choi, I. S.; Gibson, B. C.; Reineck, P.; Caruso, F. The Biomolecular Corona in 2D and Reverse: Patterning Metal-Phenolic Networks on Proteins, Lipids, Nucleic Acids, Polysaccharides, and Fingerprints. Adv. Funct. Mater. 2020, 30, 1905805.

(57) Desentis-Mendoza, R. M.; Hernández-Sánchez, H.; Moreno, A.; Rojas del C, E.; ChelGuerrero, L.; Tamariz, J.; Jaramillo-Flores, M. E. Enzymatic Polymerization of Phenolic Compounds Using Laccase and Tyrosinase from Ustilago maydis. Biomacromolecules 2006, 7, $1845-1854$.

(58) Chen, Z.; Wang, C.; Chen, J.; Li, X. Biocompatible, Functional Spheres Based on Oxidative Coupling Assembly of Green Tea Polyphenols. J. Am. Chem. Soc. 2013, 135, 4179-4182.

(59) Sunoqrot, S.; Al-Debsi, T.; Al-Shalabi, E.; Hasan Ibrahim, L.; Faruqu, F. N.; Walters, A.; Palgrave, R.; Al-Jamal, K. T. Bioinspired Polymerization of Quercetin to Produce a CurcuminLoaded Nanomedicine with Potent Cytotoxicity and Cancer-Targeting Potential in Vivo. ACS Biomater. Sci. Eng. 2019, 5, 6036-6045.

(60) Kim, Y.-J.; Chung, J. E.; Kurisawa, M.; Uyama, H.; Kobayashi, S. Regioselective Synthesis and Structures of (+)-Catechin-Aldehyde Polycondensates. Macromol. Chem. Phys. 2003, 204, $1863-1868$.

(61) Wei, J.; Wang, G.; Chen, F.; Bai, M.; Liang, Y.; Wang, H.; Zhao, D.; Zhao, Y. Sol-Gel Synthesis of Metal-Phenolic Coordination Spheres and Their Derived Carbon Composites. Angew. Chem. Int. Ed. 2018, 57, 9838-9843.

(62) Luo, W.; Xiao, G.; Tian, F.; Richardson, J. J.; Wang, Y.; Zhou, J.; Guo, J.; Liao, X.; Shi, B. Engineering Robust Metal-Phenolic Network Membranes for Uranium Extraction from Seawater. Energy Environ. Sci. 2019, 12, 607-614.

(63) Sahiner, N. One Step Poly(quercetin) Particle Preparation as Biocolloid and its Characterization. Colloids Surf., A 2014, 452, 173-180.

(64) Pranantyo, D.; Xu, L. Q.; Neoh, K. G.; Kang, E. T.; Ng, Y. X.; Teo, S. L. Tea Stains-Inspired Initiator Primer for Surface Grafting of Antifouling and Antimicrobial Polymer Brush Coatings. Biomacromolecules 2015, 16, 723-732.

(65) Xu, L. Q.; Neoh, K.-G.; Kang, E.-T. Natural Polyphenols as Versatile Platforms for Material Engineering and Surface Functionalization. Prog. Polym. Sci. 2018, 87, 165-196.

(66) Ren, P. F.; Yang, H. C.; Liang, H. Q.; Xu, X. L.; Wan, L. S.; Xu, Z. K. Highly Stable, ProteinResistant Surfaces via the Layer-by-Layer Assembly of Poly(sulfobetaine methacrylate) and 
Tannic Acid. Langmuir 2015, 31, 5851-5858.

(67) Gao, Z.; Zharov, I. Large Pore Mesoporous Silica Nanoparticles by Templating with a Nonsurfactant Molecule, Tannic Acid. Chem. Mater. 2014, 26, 2030-2037. 